\title{
CONSTRUCCIÓN DE VALORES EN LOS PROCESOS DE EMANCIPACIÓN JUVENIL
}

\author{
Bernat Albaigés Blasi ${ }^{1}$ \\ Universidad Autónoma de Barcelona
}

Remisión Artículo: 1-9-2007

Palabras Clave: Emancipación juvenil, trabajo, sociedad del conocimiento

Resumen: El presente artículo reflexiona sobre la relación existente entre los valores y las condiciones de vida de los jóvenes, haciendo especial atención a los procesos actuales de emancipación juvenil. La precarización de la experiencia laboral para muchos jóvenes ha supuesto un cambio en la forma como tradicionalmente se concebía la juventud, como etapa de transición hacia la vida adulta. La etapa juvenil se alarga en el tiempo, y también se revaloriza: deja de ser concebida como un mero tránsito hacia las 'metas' propias del adulto juventud como proceso a partir del cual se transita hasta conseguir un trabajo consistente, formar una familia, etc.-, y pasa a ser vivida como una etapa deseable por si misma, definida por sus propios valores y características. Vivir el presente, autorregularse, sentirse libre 0 reinventarse continuamente son algunos de los valores que articulan muchas identidades juveniles en la actualidad. Cabe destacar que estos son valores funcionales y adaptativos con la precariedad de los procesos de emancipación juvenil actuales, y que también, al mismo tiempo, contribuyen a normalizar y a hacer sostenible la misma experiencia de la precariedad emancipatoria. Además, estos mismos valores que definen la juventud actualmente también esconden la dificultad de trascender, cuando se quiera, la experiencia del 'ser joven'.

\section{Introducción}

Los diversos cambios de carácter económico, político, tecnológico y cultural que las sociedades modernas están experimentando en la actualidad están transformando tanto el contexto social en el que los jóvenes construyen su experiencia cotidiana como la forma en la que ésta es vivida y valorada por ellos. La globalización, el desarrollo de la sociedad del conocimiento, la extensión de las nuevas tecnologías o la penetración de las industrias del ocio, por poner algunos ejemplos, han tenido importantes repercusiones en las condiciones y estilos de vida y también en las identidades juveniles. En este sentido, y como aspecto fundamental, cabe destacar también el carácter transformador que ha supuesto la precarización del trabajo para la realidad y subjetividad juveniles.

Antes de empezar, conviene hacer una advertencia. La flexibilidad y la laxitud de las formas de vinculación social características de los tiempos actuales han contribuido a diversificar enormemente las experiencias de los jóvenes en los ámbitos del estudio, el trabajo, el tiempo libre, el consumo, la familia, etc. Si las condiciones de vida y los valores asociados a éstos son cada vez más diversos y menos coincidentes, eso significa que muchos de los jóvenes tampoco coincidirán con el conjunto de reflexiones que aquí se presentan. Más que explicar realidades compartidas por el conjunto de jóvenes, el presente artículo intenta aportar elementos de reflexión sobre determinadas realidades emergentes.

\footnotetext{
${ }^{1}$ Persona de contacto Bernat Albaigés, correo:balbaigesoc.edu
} 


\section{La precariedad como contexto de inserción laboral de los jóvenes}

El modelo keynesiano-fordista imperante hasta la II Guerra Mundial y que estaba asociado al modelo de crecimiento económico vigente hasta la década de los setenta, institucionalizó cinco grandes derechos laborales: pleno empleo garantizado por el Estado, seguridad en la renta (salarios mínimos), seguridad en la ocupación a través de normas de contratación y despido, seguridad en las condiciones de trabajo por medio de normas de seguridad, higiene y jornada, y derechos sociales a través de la Seguridad Social. Estos eran los principios normativos entorno a los cuales se regía el mundo del trabajo.

La crisis económica del 73 supuso un efecto destructivo sobre el empleo y el mercado fue incapaz de generar puestos de trabajo para toda la población activa. La aparición y consolidación del paro estructural hizo evidente el fracaso del principal objetivo entorno al que giraba el modelo económico keynesiano-fordista, el del pleno empleo. Este factor, junto con los cambios que se han producido en diversas esferas a caballo entre el siglo XX y principios del XXI han hecho temblar los pilares normativos que socialmente se habían consensuado como adecuados para el empleo.

En las sociedades occidentales, el contexto económico global, competitivo e incierto, reclama insistentemente, desde un posicionamiento ideológico neoliberal, la desregulación de la actividad económica y la lucha contra sus rigideces, con exigencias crecientes hacia la flexibilización, la minimización del intervencionismo estatal y sindical, la individualización de las relaciones laborales, etc. La amenaza de una deslocalización masiva que afecte gravemente a los mercados laborales occidentales (junto con la creciente competencia internacional, que invade de incerteza y exigencia el espacio económico e impone reducciones de los costes de producción) ha forzado a los estados a ofrecer condiciones mas beneficiosas a las empresas nacionales - para evitar la huida- y extranjeras - para atraer las inversiones -. El problema del paro se utiliza para demostrar la ineficiencia del sistema anterior, que hace necesaria la generación de trabajo bajo cualquier condición y que justifica política y socialmente las acciones encaminadas a dinamizar el mercado de trabajo mediante la supresión de sus rígidas estructuras (salarios, contratación, despidos, etc.).

En esta línea, las condiciones laborales y el empleo han devenido en elementos más de mercadeo, como un factor de producción que hay que manipular para mejorar la competitividad y la productividad de la economía. Las reivindicaciones de los sectores empresariales en las sociedades occidentales van dirigidas a la eliminación de la protección de la ocupación, la reducción de los costes laborales no salariales, el desmantelamiento de los salarios mínimos, la descentralización de la negociación de los convenios a nivel de empresa, la ampliación de las diferencias salariales, la reducción del poder de los sindicatos o la rebaja de los impuestos sobre la renta.

El resultado de este proceso ha sido la generalización de la flexibilización como una forma natural y necesaria de dirigir la esfera económica y sociolaboral y la difusión de una concepción de trabajo como un coste de producción que ha de ser reducido a la mínima expresión.

La generalización de la flexibilidad laboral que se ha promovido desde los sectores empresariales como recursos para reducir los costes laborales y optimizar así el rendimiento de la actividad productiva ha comportado una degradación de las condiciones laborales de los trabajadores en un número creciente de puestos, especialmente cuando están ubicados en la periferia del proceso productivo. Los jóvenes, precisamente, por su poca experiencia laboral tienden a ocupar estos lugares más periféricos y más vulnerables desde la perspectiva de la precarización de las condiciones laborales. 
Así es como la crisis de la norma social de la ocupación ha trasformado enormemente el escenario que el joven se ve obligado a afrontar en su proceso de inserción laboral. Se creía que la flexibiliación tendría que favorecer las facilidades de los jóvenes para incorporarse al mercado laboral. Lo cierto es que, efectivamente, existe un aumento de la creación de puestos de trabajo y, por tanto, una mayor posibilidad que esta creación se traduzca en mayores oportunidades de encontrar trabajo. Ahora bien, los puestos que se ofrecen están sujetos a una elevada rotación y son a menudo poco cualificados, mal remunerados, sin una adecuada protección legal y sin perspectivas de promoción; en definitiva, no siempre conforman una experiencia laboral positiva desde el punto de vista que asegure o, al menos, favorezca su permanencia en el mercado laboral.

En el ámbito de la sociología del trabajo se han elaborado múltiples aproximaciones teóricas al fenómeno de la precariedad. El modelo teórico elaborado por Cano (1997), uno de los más prolíficos por su claridad, concibe la precariedad en base a las limitaciones que tiene el trabajador para planificar y controlar su presente y futuro y la consecuente vulnerabilidad social y económica que ésta representa para su vida. Según este autor, esta precariedad se concreta en función básicamente de cuatro dimensiones fundamentales.

La primera gira entorno a la inseguridad que manifiesta el trabajador sobre la continuidad de la relación laboral. La reducción de los costes de despido hace que esta dimensión no afecte exclusivamente a los trabajadores temporales. La precariedad aparece cuando el futuro de una situación no está asegurado y se manifiesta en forma de inestabilidad y de deterioro de la salud mental (ansiedad, ausencia de autoestima, etc.), pero también en una pérdida gradual de la empleabilidad. Estos son los elementos que pueden incidir negativamente sobre el proceso de inserción sociolaboral del joven. La temporalidad y la elevada rotación laboral que caracteriza las experiencias laborales de los jóvenes, con excesivas variaciones de contenidos y tareas, de compañeros, de ambientes y de normas, deriva en "una mayor dificultad para la adaptación en el trabajo (...), en una degradación de las condiciones de trabajo y en las dificultades para articular un red de relaciones alrededor del mundo laboral" (Miró, 1999:38).

La segunda gira entorno a la degradación y la vulnerabilidad de la situación de trabajo. La vulnerabilidad se manifiesta en una pérdida de control sobre la propia situación laboral y tiene unos efectos nefastos sobre la seguridad e higiene en el espacio de trabajo, la posición del trabajador dentro de la organización productiva, la duración y la distribución de la jornada, la cualidad del suporte relacional y técnico, las posibilidades de promoción y de formación, etc. El trabajador, en la lógica de la flexibilidad, pierde poder y se encuentra más que nunca en manos del empresario y de la lógica del mercado. La incertidumbre de la situación actúa como un instrumento coercitivo en pro del rendimiento del trabajador y de la productividad de la empresa y en contra de las posibilidades del trabajador para exigir unas determinadas condiciones laborales. Esta tendencia se acentúa especialmente en el caso del joven, por su situación intrínsecamente vulnerable y débil dentro del mercado laboral.

La tercera gira entorno a la insuficiencia e incertidumbre de los ingresos salariales. Esta dimensión de la precariedad tendría unos efectos dramáticos sobre la subsistencia del trabajador. "El nivel de retribución determina en buena medida el nivel de vida, la posición social del trabajador e incluso su propia percepción sobre la calidad de la ocupación" (Cano, 1997:82). La inestabilidad y la elevada rotación de los puestos de trabajo no permiten al trabajador planificar el futuro ya que no tiene garantizada una continuidad en los ingresos. Para los jóvenes, esta dimensión se puede ver atenuada por la familia, que se convierte en "el colchón de esta disfuncionalidad, permitiendo la prolongación de la vida adolescente" (Cano, 1997:87).

Y, finalmente, la cuarta hace referencia a la insuficiencia y reducción de la protección social. La protección social aumenta el control y las garantías de subsistencia en el futuro y, por tanto, su 
reducción se traduce en incertidumbre y vulnerabilidad para el trabajador. Como apunta Miró (1999:42), esta dimensión se ha acentuado no tan solo por una reducción efectiva de la protección social en los trabajos estables, sino también por el desarrollo de la contratación temporal y a tiempo parcial, que conduce a los colectivos más débiles a no poder disfrutar de las ventajas de la protección social ya que se produce "una discriminación indirecta por la necesidad de cubrir determinados períodos de tiempo para acceder a las prestaciones".

\section{La precariedad como contexto de transición a la vida adulta}

Anteriormente hemos comentado que la precariedad laboral transforma la experiencia de la juventud, del ser y sentirse joven. Entre otros aspectos, cabe destacar que la precariedad laboral ha puesto en crisis los modelos tradicionales de emancipación juvenil.

Las numerosas y diversas definiciones sobre la juventud que las ciencias sociales han aportado coinciden en destacar su condición de transición social hacia la vida adulta. Desde este punto de vista, el período juvenil está definido socialmente por una meta: la integración plena del individuo en la sociedad por medio del trabajo y por la autonomía en relación con la familia de origen, características propias de la adultez.

La juventud, como transición a la vida adulta, consiste en un proceso de emancipación laboral, domiciliaria y familiar. "Emanciparse significa liberarse de cualquier clase de dependencia, que en el caso de los y las jóvenes se concreta en el paso de la dependencia de los padres a la 'libertad' de la vida adulta. La emancipación se puede entender como un proceso extensivo en el tiempo que comporta la independencia económica -normalmente asociada a la transición escuela-trabajo-, el dominio de un espacio que se usa como espacio propio -transición domiciliaria- y la formación de una familia -transición familiar. Esto es, la emancipación sería el fruto de lo que se denomina 'transición hacia la vida adulta'" (López, 2006:136).

Respecto del proceso de emancipación laboral, la precariedad ha provocado que los modelos de transición tradicionales, basados en trayectorias de inserción hacia el mundo del trabajo más o menos continuas y lineales, con una cierta correspondencia entre la formación y la cualificación profesional, hayan dado lugar a transiciones caracterizadas por las discontinuidades y por la relación discontinua entre los ámbitos educativo y laboral. La escuela resulta ser solamente la primera etapa en trayectorias complejas y menos directas, que sólo en ocasiones resultan ser continuas y en el resto de las ocasiones se caracterizan por avances y retrocesos consignados con vueltas al sistema educativo o con periodos de espera, que conducen a los jóvenes a diferentes e imprevisibles posiciones dentro de la estructura social y ocupacional.

Estas transiciones corresponderían a las denominadas por Casal $(1997,2004)$ como trayectorias de precariedad, caracterizadas por una ruptura real de la linealidad imperante en los modelos característicos de etapas anteriores. A diferencia de otras modalidades transicionales en retroceso, las trayectorias en precariedad consisten en que "la inserción en el trabajo queda dominada por la rotación laboral, el paro, la subocupación y, en definitiva, la plena inestabilidad, sin conseguir configurar en sus sucesivas entradas y salidas del mundo laboral una orientación clara hacia la obtención de expectativas, sean estas altas o bajas. La inestabilidad es tal que los individuos se ven incapaces de generar cualquier tipo de desarrollo de carrera laboral o profesional con una cierta coherencia" (Albaigés, Sisto y Román, 2003:78).

Esta incertidumbre y dificultad para decidir y controlar la propia situación de existencia también se pone de manifiesto en los procesos de emancipación domiciliaria. La precariedad laboral limita las posibilidades de subsistencia económica y, consecuentemente, de lograr o desarrollar todo aquello que acarree gasto. Como es sabido, esa dificultad se añade al elevado precio de 
la vivienda y de los alquileres, lo cual, sumado, provoca que muchos jóvenes tengan que permanecer en casa de los progenitores hasta edades avanzadas o, en caso contrario, de verse obligados a idear soluciones imaginativas y de circunstancias para poder vivir fuera. Así pues, la precariedad también se evidencia en las dificultades y en la situación de provisionalidad que experimenta el joven en el proceso de abandono del hogar de origen y de formación de un hogar independiente.

Precisamente, la precariedad laboral y las dificultades relacionadas con la vivienda hacen que los jóvenes tampoco tengan el control sobre los procesos de emancipación familiar. A menudo, especialmente entre las clases medias, son los mismos progenitores los que dan apoyo económico a los hijos para que puedan ampliar su formación a pesar de residir fuera de casa, los que ponen a su nombre los contratos de alquiler de viviendas de sus hijos, los avalistas de préstamos, etc. (López, 2006). A pesar de que cuando viven en casa la familia de origen se habla de autonomía relativa para referirse a la capacidad creciente de maniobra y de libertad de elección de los jóvenes en muchos campos de acción (Casal et al., 2004), la dependencia se prolonga en el tiempo, incluso tras la conformación de un núcleo familiar propio e "independiente".

Esta dependencia material es la principal evidencia de la precariedad que experimentan los jóvenes en sus procesos de emancipación. Como comentaba Cano (1997) para el ámbito laboral, la precariedad en los procesos de emancipación se pone de manifiesto con limitaciones a la hora de planificar y controlar su presente y futuro y la consecuente vulnerabilidad social y económica que esta representa para su vida. En otras palabras, Feixa (2001) habla que vivir la juventud consiste en "experimentar lo errático del destino incierto. Para los jóvenes de hoy, eso significa migrar por diversos ecosistemas geográficos y sociales, cambiar los roles sin cambiar el estatus, recorrer mundo y volver periódicamente a casa de los padres, hacerse adulto y volver a la juventud cuando el trabajo se acaba, disfrazarse de joven cuando ya se está casado y se gana lo mismo que un adulto, etc.".

\section{Condiciones materiales de vida como generadores de valores: la valoración de la juventud.}

Estas dificultades de emancipación han hecho alargar las transiciones, y la etapa juvenil, perpetuada, adopta nuevas formas y nuevos sentidos. Si tradicionalmente se había caracterizado como un proceso de transición hacia la adultez, en el que ser joven suponía estar en una especie de tránsito "carencial" en relación con la consecución de las metas propias del adulto (emancipación laboral, residencial y familiar), en la actualidad la juventud tiende a ser una etapa deseable por si misma, definida por sus propias características y valores, ya no como una mera etapa de paso, sino como una estabilidad revalorizada (paradójicamente).

A pesar de la precariedad en los procesos de emancipación, esta estabilidad es posible porque la asunción de responsabilidades adultas se pospone en el tiempo (trabajo estable, constitución del hogar familiar, procreación, etc.). De hecho, la misma prolongación de la juventud es una forma de garantizar la estabilidad material y social a un grupo social que cuenta con dificultades objetivas de autosostenerse y de proyectarse autónomamente hacia el futuro.

Paralelamente, esta estabilidad se revaloriza porque encuentra espacios, como el del tiempo libre, que, lejos de proyectos adultos, permiten alimentar de contenidos este tiempo vital, permiten convertir la accidentalidad del proceso juvenil en algo sustancial (Martínez i Pérez, 1998). El ocio se ha convertido en un espacio de creación cultural y de identidad típicamente juvenil: el joven puede construir, descubrir y expresar su propia identidad, sin limitaciones aparentes. 
La ausencia de responsabilidades adultas sitúa en la libertad y en la ausencia de vínculos uno de los principales valores de la juventud. Las oportunidades de autorregulación que ofrecen estos espacios contrastan con la heterorregulación característica de los espacios propiamente adultos (familiar, laboral, etc.) o contralados por adultos (como el escolar). En la esfera del ocio, Nothing is imposible, Just do it, (como proclaman los eslóganes de Adidas y Nike): las posibilidades son infinitas, nosotros decidimos si queremos hacerlo. La búsqueda de autenticidad en los estilos de vida, el relativismo moral en relación con las normas y convenciones sociales, la voluntad de que los tiempos de obligaciones (trabajo, estudios, etc.) no interfieran en los tiempos de ocio, el reclamo de mayores niveles de autonomía respecto de los progenitores, etc. son manifestaciones diferentes del valor de la libertad para los jóvenes. La existencia de un mercado que es capaz de satisfacer las necesidades de manera inmediata alimenta la ilusión por la autorregulación.

Incluso, ante situaciones que coarten objetivamente las oportunidades de proyección en el futuro, como es el caso de la precariedad laboral, pueden llegar a hacer aparecer entre algunos jóvenes (con una situación de estabilidad material y social garantizada) interpretaciones de la propia situación laboral en clave de oportunidades de libertad (Albaigés, Sisto i Román, 2003). "Después de una reacción de frustración provocada por la disonancia entre los modelos sociolaborales para los cuales han sido socializados (estabilidad contractual) y los que encuentran en la propia experiencia cotidiana (desocupación, precariedad laboral, etcétera), algunos jóvenes tienden a normalizar con sus discursos la precariedad como una situación natural y necesaria para la incorporación definitiva a la vida activa 'propia' del adulto, estable y confortable. Son estrategias adaptativas que se caracterizan por una valoración neutral de las condiciones de empleo situada en el presente (y en la que la problematización se pospone amparado por el 'todavía soy joven') o, incluso, por una valoración positiva, en términos de libertad ('estar menos vinculado al trabajo') y de oportunidades de aprovechar mejor otras esferas vitales más significativas para el joven".

Ahora bien, esta valoración de libertad forma parte de una ilusión, de una realidad virtual (si tomamos como metáfora la sensación que nos generan las TICs de poder controlar mejor y de forma inmediata las informaciones y las relaciones sociales claves, cuando son, a menudo, estas tecnologías las que nos controlan a nosotros). Se trata de una experiencia de libertad muy centrada en el presente y en el yo, y en cambio la libertad sobre aquello que se proyecta hacia el futuro o hacia los otros se diluye. Podríamos decir que "somos libres de decidir sobre aquello que podemos decidir".

En primer lugar, la incertidumbre sobre aquello que sucederá mañana (como consecuencia de la precariedad en las condiciones de vida del presente), así como la postergación de la asunción de las responsabilidades propiamente adultas (que hacen menos necesarias la acomodación del presente respecto a determinados proyectos de futuro), refuerzan determinadas actitudes presentistas entre los jóvenes. Diversos trabajos (Casal y otros, 2004, Estradé y otros, 2002, Secarrant, 2001, etc.) han insistido recientemente sobre esta cuestión con relación a los jóvenes catalanes. La mayoría de los jóvenes declara vivir al día y que esta actitud se acentúa entre los más jóvenes y entre los que parten de un estatus económico más precario. Como más incierto es el futuro, más se acentúan las actitudes focalizadas en el presente.

Los cambios constantes en el entorno, la reinvención continua de nuestros deseos y expectativas (en un contexto de reinvención constante de las instituciones, como apunta Sennett -2000-), los comportamientos flexibles condicionados a estos cambios, etc., no ofrecen oportunidades de planificar y comprometerse en proyectos de futuro, porqué las cosas pueden cambiar en el futuro inmediato y pueden ser diferentes de lo que habíamos pensado en el presente. 
$Y$ en segundo lugar, esta incertidumbre sobre el futuro inmediato, que hace imprevisible nuestra situación y también la de los otros, dificulta proyectarnos en los otros. De hecho, las discontinuidades y los cambios constantes en las transiciones de los jóvenes contribuyen a diversificar sus condiciones materiales de vida (respecto sus experiencias laborales, formativas, familiares, etc.). Esta fragmentación dificulta la posibilidad de encontrar o articular relatos compartidos entre los propios jóvenes ("hay historia, pero no hay narrativa compartida de dificultad y, por tanto, no hay destino compartido" -Sennett, 2000:154- ). La prevalencia de la autorregulación, centrada en las propias necesidades inmediatas y con miedo a contraer vínculos y dependencias que después no se puedan mantener, así como la ausencia de relatos compartidos, fomentan una cierta individualización (e individualismo) de los procesos de emancipación. Ante la precariedad de estos procesos, la seguridad se consigue evitando la asunción de compromisos y restringiendo las exigencias que la sociedad aboga hacia nosotros. Los proyectos colectivos, las vinculaciones sociales, se producen cuando y mientras los diferentes intereses y experiencias particulares coinciden en un mismo punto.

En este sentido, Petras (citado en Obeso, 1997) señala que la individualización se orienta hacia la "autorrealización" y la "búsqueda de la felicidad personal", una felicidad personal de carácter mas bien acomodaticio que tiende a desvincularse de los vínculos sociales de integración cooperativa. "Valores y sistemas de significación se han hecho ambiguos, generales y sin obligar a compromisos, lo que origina una mentalidad centrada en el yo, que no se vincula ni se compromete con ningún sistema. La disminución del interés por la res pública es una consecuencia de eso, al mismo tiempo que unas crecientes aspiraciones a la 'propia identidad' y 'autenticidad'” (Petras, citado en Obeso, 1997:36).

En definitiva, la posición de la problematización sobre la proyección de futuro y la individualización con la que los jóvenes viven los procesos de emancipación alimenta una cierta ilusión de libertad y estabilidad.

La focalización en el presente y la posposición de la toma de decisiones sobre la proyección de futuro (pensar en "¿qué seré de mayor?") no significa que el "ideal" de adultez no exista, de que los jóvenes no tengan aspiraciones a tener un trabajo estable, una vivienda estable, una familia estable, etc. Sin embargo, los jóvenes son conscientes de que su situación actual no conduce a ninguno de estos futuros. "Los nuevos modelos culturales ponen en evidencia una auténtica ruptura entre ser joven -hoy- y ser adulto - mañana-, de tal forma que la normalización transitoria de la precariedad emancipatoria tendría la función de atenuar sus efectos, sin perder la esperanza de un cambio futuro en las oportunidades - y no en el ideal de madurez (que permanece inmutable)" (Albaigés, Sisto y Román, 2003).

\section{Valores como reproductores de las condiciones materiales de vida: la normalización de la precariedad.}

La precariedad de los procesos de emancipación tiene para el joven un carácter provisional, que ha de ser superada por oportunidades que se ajusten a sus sueños de adultez: es decir, la contradicción presente entre las dificultades de acceso al trabajo o a la vivienda estable y "el ideal de adultez" llevan al joven a considerar las dificultades en el momento presente como tal, una cosa que cambiará con el tiempo. "La normalización de la situación presente actúa como una normalización de la anomalía, es decir, en el límite sutil entre 'lo que vive es anormal y, por tanto, se normalizará' y 'esta anomalía es normal para el actual periodo de mi vida'. Esto, por un lado, favorece una actitud de aceptación pasiva y, por la otra, remarca la presencia de una valoración de la situación presente que se presta a construir esta normalización de la anomalía" (Albaiges, Sisto y Román, 2003). 
Así se cierra el círculo de precariedad: la provisionalidad de los procesos de emancipación se soporta porque se construyen valores que diluyen el futuro y hacen posible la experiencia de la provisionalidad sin frustraciones traumáticas en el presente. Así se abren espacios de legitimación de la propia precariedad.

A pesar del riesgo de no "tomarse a sí mismo en serio porque siempre es consciente que los términos en que se describen están sujetos al cambio, porque siempre es consciente de la contingencia y la fragilidad de su vocabulario final y, por tanto, de sí mismo" (a Sennett, 2000:122), esta asunción de la provisionalidad como una circunstancia normal en el presente resulta absolutamente funcional con los modelos sociolaborales y de acceso a la vivienda actuales, que no les ofrece oportunidades de insertarse en el mundo adulto de una manera estable. Afirmar que el joven normaliza la precariedad en términos de libertad y de independencia no significa que prefiera esta situación a la estabilidad en la emancipación.

Adicionalmente, la construcción de la juventud se fundamenta en modelos culturales juveniles que a menudo anteponen la autorregulación o la satisfacción de las necesidades inmediatas como un principio fundamental, porque están adaptados al contexto cultural que les ha tocado vivir, porque les permite revalorizar y dar significado a la propia experiencia. Así, tampoco significa que estos modelos culturales sean coincidentes con la precariedad, en el sentido de reforzar al joven en una situación precaria, sino que a menudo ni se contraponen ni entran en conflicto -siempre que el joven tenga asegurados los recursos necesarios para vivir a través del trabajo (precario) o de la familia-.

Por tanto, la revalorización de la juventud no ha de servir, bajo discursos de una cierta acomodación juvenil, para justificar o encubrir el incremento de la precariedad en el paso de joven a adulto, especialmente porque existen personas que quieren y no pueden iniciar procesos de emancipación familiar, laboral y residencial. Es en estos casos cuando se hace evidente que la precariedad representa un potente generador de desigualdad social.

Subrayar la importancia de la subjetividad y su incidencia en la consolidación de la precariedad no significa que esta sea la responsable de la situación del joven en el mundo laboral. Cabe añadir que el desarrollo de acciones estratégicas encaminadas a la obtención de un proyecto de vida por parte de los individuos sin las herramientas contextuales necesarias pueden llevar a la frustración. La salida del círculo de la precariedad, si bien se resuelve en la esfera subjetiva del individuo, depende intrínsecamente de las políticas socioeconómicas y también culturales que adquieren formas concretas y que conforman el contexto de los individuos. Por eso, es necesario que estas sean sensibles a la fragilidad de los estilos de vinculación social colectiva que acompañan las diferentes maneras de flexibilización, que se plantean nuevas políticas que eviten la precariedad del trabajo y, también y sobre todo, que promuevan verdaderos procesos de integración social. 


\section{Referencias bibliográficas}

Albaigés, B., Sisto, V. i Román, J.A. (2003). Crisi del treball i emergència de noves formes de subjectivitat laboral en els joves. Col.lecció Estudis núm. 10. Barcelona: Secretaria General de Joventut.

Cano, E. (1997): Canvi socioeconòmic i precarització laboral en el sistema capitalista. Tesi doctoral. Universidad de Valencia.

Casal, J. (1996): "Modos emergentes de transición a la vida adulta en el umbral dels siglo XXI: aproximación sucesiva, precariedad y desestructuración". Revista REIS, núm. 75, pàg 295-317.

Casal, J et alt. (2004). Enquesta als joves de Catalunya 2002. Col-lecció Estudis núm. 13. Barcelona: Secretaria General de Joventut.

Estradé, i altres (2003). Joves i valors. Els joves catalans en l'Enquesta Europea de Valors. Col.lecció Estudis núm. 9. Barcelona: Secretaria General de Joventut.

Feixa, C. (2001). Generació @. La joventut al segle XXI, Col-lecció Aportacions núm 12. Barcelona: Secretaria General de Joventut.

López, J. (2006). Habitatge. A Informe sobre la joventut al 2005. Observatori Català de la Joventut. Barcelona: Secretaria General de Joventut

Martínez, R. i Pérez, J. (1997). El gust juvenil en joc. Barcelona: Diputació de Barcelona.

Miró, I. i Ortiz de Villacian, D. (1999): Treball, valors i canvi. Les ruptures en la precarietat. Barcelona: Fundació Serveis de Cultura Popular.

Obeso, C. (1997): Desempleo y precarización del mercado de trabajo. A. Pérez M. et al. (ed.), El trabajo en el futuro. Bilbao: Ediciones Deusto.

Sennett, R. (2000): La corrosión del carácter. Las consecuencias personales del trabajo en el nuevo capitalismo. Barcelona: Anagrama.

Serracant, P. (2001). Viure al dia. Condicions d'existència, comportaments i actituds dels joves catalans. Informe sobre la joventut catalana al 2001. Col·lecció Estudis núm. 6. Barcelona: Secretaria General de Joventut. 
Architecture, City, and Environment

Arquitectura, Ciudad y Entorno 